CUBO A Mathematical Journal

Vol.12, $N^{\underline{O}}$ 02, (145-167). June 2010

\title{
On homogeneous polynomial solutions of generalized Moisil-Théodoresco systems in Euclidean space
}

\author{
Richard Delanghe \\ Department of Mathematical Analysis, \\ Clifford Research Group, \\ Ghent University, \\ Galglaan 2, B-9000 Ghent, Belgium
}

\begin{abstract}
Let for $s \in\{0,1, \ldots, m+1\}(m \geq 2), \mathbb{R}_{0, m+1}^{(s)}$ be the space of s-vectors in the Clifford algebra $\mathbb{R}_{0, m+1}$ constructed over the quadratic vector space $\mathbb{R}^{0, m+1}$ and let $r, p, q, \in \mathbb{N}$ be such that $0 \leq r \leq m+1, p<q$ and $r+2 q \leq m+1$. The associated linear system of first order partial differential equations derived from the equation $\partial_{x} W=0$ where $W$ is $\mathbb{R}_{0, m+1}^{(r, p, q)}=\sum_{j=p}^{q} \oplus \mathbb{R}_{0, m+1}^{(r+2 j)}$-valued and $\partial_{x}$ is the Dirac operator in $\mathbb{R}^{m+1}$, is called a generalized Moisil-Théodoresco system of type $(r, p, q)$ in $\mathbb{R}^{m+1}$. For $k \in N, k \geq 1, M T^{+}(m+$ $1 ; k ; \mathbb{R}_{0, m+1}^{(r, p, q)}$ ), denotes the space of $\mathbb{R}_{0, m+1}^{(r, p, q)}$-valued homogeneous polynomials $W_{k}$ of degree $k$ in $\mathbb{R}^{m+1}$ satisfying $\partial_{x} W_{k}=0$. A characterization of $W_{k} \in M T^{+}\left(m+1 ; k ; \mathbb{R}_{0, m+1}^{(r, p, q)}\right)$ is given in terms of a harmonic potential $H_{k+1}$ belonging to a subclass of $\mathbb{R}_{0, m}^{(r, p, q)}$-valued solid harmonics of degree $(k+1)$ in $\mathbb{R}^{m+1}$. Furthermore, it is proved that each $W_{k} \in$ $M T^{+}\left(m+1 ; k ; \mathbb{R}_{0, m+1}^{(r, p, q)}\right)$ admits a primitive $W_{k+1} \in M T^{+}\left(m+1 ; k+1 ; \mathbb{R}_{0, m+1}^{(r, p, q)}\right)$. Special attention is paid to the lower dimensional cases $\mathbb{R}^{3}$ and $\mathbb{R}^{4}$. In particular, a method is developed for constructing bases for the spaces $M T^{+}\left(4 ; k ; \mathbb{R}_{0,4}^{(r, p, q)}\right), r$ being even.
\end{abstract}

\section{RESUMEN}

Para $s \in\{0,1, \ldots, m+1\}(m \geq 2), \mathbb{R}_{0, m+1}^{(s)}$ el espacio de los s-vectors en el algebra de Clifford $\mathbb{R}_{0, m+1}$ construida sobre el espacio de vectores cuadráticos $\mathbb{R}^{0, m+1}$ sea $r, p, q, \in \mathbb{N}$ tal que $0 \leq r \leq m+1, p<q$. El sistema lineal asociado de ecuaciones diferenciales 
parciales de primer orden derivado de la ecuación $\partial_{x} W=0$ donde $W$ es $\mathbb{R}_{0, m+1}^{(r, p, q)}=$ $\sum_{j=p}^{q} \oplus \mathbb{R}_{0, m+1}^{(r+2 j)}$-valuada y $\partial_{x}$ es el operador de Dirac en $\mathbb{R}^{m+1}$, es llamado un sistema de Moisil-Théodoresco generalizado de tipo $(r, p, q)$ en $\mathbb{R}^{m+1}$. Para $k \in N, k \geq 1, M T^{+}(m+$ $1 ; k ; \mathbb{R}_{0, m+1}^{(r, p, q)}$, denota el espacio de polinomios homogéneos $W_{k} \mathbb{R}_{0, m+1^{-}}^{(r, p, q)}$ valuados de grado $k$ en $\mathbb{R}^{m+1}$ satisfaciendo $\partial_{x} W_{k}=0$. Una caracterización de $W_{k} \in M T^{+}\left(m+1 ; k ; \mathbb{R}_{0, m+1}^{(r, p, q)}\right)$ es dada en términos de un potencial armónico $H_{k+1}$ perteneciendo a una subclase de armónicos consistentes $\mathbb{R}_{0, m}^{(r, p, q)}$-valuados de grado $(k+1)$ in $\mathbb{R}^{m+1}$. Además es probado que todo $W_{k} \in M T^{+}\left(m+1 ; k ; \mathbb{R}_{0, m+1}^{(r, p, q)}\right)$ admite una primitiva $W_{k+1} \in M T^{+}(m+1 ; k+$ $\left.1 ; \mathbb{R}_{0, m+1}^{(r, p, q)}\right)$. Una especial atención es dada a los casos de dimensión baja $\mathbb{R}^{3} \mathrm{y} \mathbb{R}^{4}$. En particular, un metodo es desarrollado para construir bases para espacios $M T^{+}\left(4 ; k ; \mathbb{R}_{0,4}^{(r, p, q)}\right), r$ siendo par.

Key words and phrases: Clifford analysis; Moisil-Théodoresco systems; conjugate harmonic funtions; harmonic potentials; polynomial bases

Mathematics Subject Classification (2000): 30G35

\section{Introduction}

Let $\tilde{f}=\left(f_{0}, \vec{f}\right)$ with $\vec{f}=\left(f_{1}, f_{2}, f_{3}\right)$ be a $\mathbb{R}^{4}$-valued $C_{1}$-function in some oppropriate open domain $\Omega$ of $\mathbb{R}^{3}$.

In $[20]$ it was pointed out that the Riesz system

$$
(R)\left\{\begin{array}{l}
\operatorname{div} \vec{f}=0 \\
\operatorname{curl} \vec{f}=0
\end{array}\right.
$$

and the Moisil-Théodoresco system

$$
(M T)\left\{\begin{array}{l}
\operatorname{div} \vec{f}=0 \\
\operatorname{grad} f_{0}+\operatorname{curl} \vec{f}=0
\end{array}\right.
$$

are examples of natural generalizations of the Cauchy-Riemann equations in the plane to Euclidean space $\mathbb{R}^{3}$.

Obviously (1.1) may be derived from (1.2) by taking $f_{0}=0$.

For the original definition of the $(M T)$-system (1.2) we refer to [17].

The (MT)-system (1.2) may also be obtained by making use of the algebra $\mathbb{H}$ of real quaternions. Indeed, consider in $\mathbb{R}^{3}$ the operator $D_{3}=i \partial_{x_{0}}+j \partial_{x_{1}}+k \partial_{x_{2}}$ where $(i, j, k)$ is the standard set of imaginary units in $\mathbb{H}$, and associate with $\left(f_{0}, \vec{f}\right)$ the $\mathbb{H}$-valued function $f=f_{0}+i f_{1}+j f_{2}+k f_{3}$. Then $\tilde{f}=\left(f_{0}, \vec{f}\right)$ satisfying $(1.2)$ is equivalent to $f$ satisfying the equation (see e.g. [15])

$$
D_{3} f=0
$$


In [14] solutions to (1.3) were called $\mathbb{H}$-regular.

In [20] the authors studied properties of solutions to more general first order linear systems in Euclidean space generalizing the Cauchy-Riemann equations and having the property that they are invariant under rotations. Among such systems figures in particular the Hodge-de Rham system

$$
(H d R)\left\{\begin{array}{l}
d \omega^{r}=0 \\
d^{*} \omega^{r}=0
\end{array}\right.
$$

$\omega^{r}$ being a smooth $r$-form.

Putting $\mathbb{R}^{m+1}=\left\{x=\left(x_{0}, \underline{x}\right): \underline{x}=\left(x_{1}, \ldots, x_{m}\right) \in \mathbb{R}^{m}\right\}$, let us recall that for a smooth $s$-form $\omega^{s}=$ $\sum_{|A|=s} \omega_{A}^{s} d x^{A}(0 \leq s \leq m+1)$ where for $A=\left\{i_{1}, i_{2}, \ldots, i_{s}\right\}$ with $0 \leq i_{1}<i_{2}<\ldots<i_{s} \leq m, d x^{A}=$ $d x^{i_{1}} \wedge d x^{i_{2}} \wedge \ldots \wedge d x^{i_{s}}, d \omega^{s}$ and $d^{*} \omega^{s}$ are defined by (see [16])

$$
d \omega^{s}=\sum_{A} \sum_{i=0}^{m} \partial_{x_{i}} \omega_{A}^{s} d x^{i} \wedge d x^{A}
$$

and

$$
d^{*} \omega^{s}=\sum_{A} \sum_{j=1}^{s}(-1)^{j \partial_{x_{i_{j}}}} \omega_{A}^{s} d x^{A \backslash\left\{i_{j}\right\}}
$$

Notice that if in (1.4), $r=1$ and $\omega^{1}=u_{0} d x^{0}+u_{1} d x^{0}+u_{1} d x^{1}+\ldots+u_{m} d x^{m}$, then

$$
\left\{\begin{array} { l } 
{ d \omega ^ { 1 } = 0 } \\
{ d ^ { * } \omega ^ { 1 } = 0 }
\end{array} \Longleftrightarrow \left\{\begin{array}{l}
\frac{\partial u_{j}}{\partial x_{i}}-\frac{\partial u_{i}}{\partial x_{j}}=0, i, j=0, \ldots, m ; i \neq j \\
\sum_{i=0}^{m} \frac{\partial u_{i}}{\partial x_{i}}=0
\end{array}\right.\right.
$$

Obviously, (1.6) generalizes to $\mathbb{R}^{m+1}$ the system $(R)$ in $\mathbb{R}^{3}$ defined by (1.1).

An $(m+1)$-system $u=\left(u_{0}, u_{1}, \ldots, u_{m}\right)$ satisfying (1.6) was called in [19] a system of conjugate harmonic funtions.

More generally, introducing the differential operator $D=d+d^{*}$, its action on the smooth differential form $\omega=\sum_{s=0}^{m+1} \omega^{s}$ in $\Omega \subset \mathbb{R}^{m+1}$ open, leads to the first order system of differential equations

$$
\left(d+d^{*}\right) \omega=0 \Longleftrightarrow\left\{\begin{array}{l}
d^{*} \omega^{1}=0 \\
d \omega^{s}+d^{*} \omega^{s+2}=0, s=0, \ldots, m-1 \\
d \omega^{m}=0
\end{array}\right.
$$

Obviously, if $\omega=\omega^{r}, 0 \leq r \leq m+1$ being fixed, the system (1.7) reduces to the Hodge-de Rham system (1.4).

In $\mathbb{R}^{3}$, associating with $\tilde{f}=\left(f_{0}, \vec{f}\right)$, the form $\omega=\omega^{0}+\omega^{2}$ with $\omega^{0}=f_{0}$ and $\omega^{2}=f_{1} d x^{1} \wedge d x^{2}+$ 
$f_{2} d x^{2} \wedge d x^{0}+f_{3} d x^{0} \wedge d x^{1}$, then clearly $\left(d+d^{*}\right) \omega=0$ is equivalent with $\left(f_{0}, \vec{f}\right)$ satisfying (1.2).

Let us now describe how the system (1.7) may also be obtained within the framework of Clifford analysis.

Let $\mathbb{R}^{0, m+1}$ be the real vector space $\mathbb{R}^{m+1}$ equipped with a quadratic form of signature $(0, m+1)$ and let $e=\left(e_{0}, e_{1}, \ldots, e_{m}\right)$ be an orthogonal basis of $\mathbb{R}^{0, m+1}$. Furthermore, let $\mathbb{R}_{0, m+1}$ be the universal Clifford algebra constructed over $\mathbb{R}^{0, m+1}$. Then $\mathbb{R}_{0, m+1}$ is a linear associative but non-commutative algebra with identity and having dimension $2^{m+1}$.

The basic multiplication rules in $\mathbb{R}_{0, m+1}$ are governed by

$$
\begin{gathered}
e_{i}^{2}=-1, i=0,1, \ldots, m \\
e_{i} e_{j}+e_{j} e_{i}=0, i \neq j, i, j=0,1, \ldots m .
\end{gathered}
$$

A basis for $\mathbb{R}_{0, m+1}$ is given by the set $\left(e_{A}: A \subset\{0,1, \ldots, m\},|A|=s, s=0,1, \ldots, m+1\right)$ where $A=\left\{i_{1}, \ldots, i_{s}\right\}$ with $0 \leq i_{1}<i_{2}<\ldots<i_{s} \leq m$ and $e_{A}=e_{i_{1}} e_{i_{2}} \ldots e_{i_{s}}, e_{\emptyset}=1$ being the identity element of $\mathbb{R}_{0, m+1}$.

For $s \in\{0,1, \ldots, m+1\}$ fixed the space $\mathbb{R}_{0, m+1}^{(s)}$ of $s$-vectors in $\mathbb{R}_{0, m+1}$ is defined by $\mathbb{R}_{0, m+1}^{(s)}=$ $\operatorname{span}_{\mathbb{R}}\left(e_{A}:|A|=s\right)$.

Obviously

$$
\mathbb{R}_{0, m+1}=\sum_{s=0}^{m+1} \oplus \mathbb{R}_{0, m+1}^{(s)}
$$

Denote by [ $]_{s}$ the projection of $\mathbb{R}_{0, m+1}$ onto $\mathbb{R}_{0, m+1}^{(s)}$.

For a 1-vector $v$ and a $s$-vector $w^{s}$, we have that $v w^{s}$ splits into

$$
v w^{s}=\left[v w^{s}\right]_{s-1}+\left[v w^{s}\right]_{s+1}
$$

where

$$
\left[v w^{s}\right]_{s-1}=\frac{1}{2}\left(v w^{s}-(-1)^{s} w^{s} v\right)
$$

and

$$
\left[v w^{s}\right]_{s+1}=\frac{1}{2}\left(v w^{s}+(-1)^{s} w^{s} v\right)
$$

Introducing the Dirac operator $\partial_{x}=\sum_{i=0}^{m} e_{i} \partial_{x_{i}}$ in $\mathbb{R}^{m+1}$, then by (1.9) its (left) action on a $\mathbb{R}_{0, m-1}^{(s)}$-valued smooth funtion $W^{s}$ in $\Omega \subset \mathbb{R}^{m+1}$ open reads:

$$
\partial_{x} W^{s}=\left(\partial_{x}^{+}+\partial_{x}^{-}\right) W^{s}
$$

where

$$
\partial_{x}^{+} W^{s}=\left[\partial_{x} W^{s}\right]_{s+1}=\frac{1}{2}\left(\partial_{x} W^{s}+(-1)^{s} W^{s} \partial_{x}\right)
$$


and

$$
\partial_{x}^{-} W^{s}=\left[\partial_{x} W^{s}\right]_{s-1}=\frac{1}{2}\left(\partial_{x} W^{s}-(-1)^{s} W^{s} \partial_{x}\right),
$$

$W^{s} \partial_{x}$ meaning that $\partial_{x}$ acts from the right on $W^{s}$.

It thus follows that if the $\mathbb{R}_{0, m+1}$ valued smooth function $W$ in $\Omega$ is decomposed along (1.8), i.e. $W=\sum_{s=0}^{m+1} W^{s}$ with $W^{s}$ smooth and $\mathbb{R}_{0, m+1}^{(s)}$-valued in $\Omega$, then

$$
\partial_{x} W=0 \Longleftrightarrow\left\{\begin{array}{l}
\partial_{x}^{-} W^{1}=0 \\
\partial_{x}^{+} W^{s}+\partial_{x}^{-} W^{s+2}=0, s=0,1, \ldots, m-1 \\
\partial_{x}^{+} W^{m}=0
\end{array}\right.
$$

Obviously, the systems (1.7) and (1.11) show some parallelism, which becomes fully clear if one considers the isomorphism $\Theta$ between the spaces $\mathcal{E}\left(\Omega ; \wedge^{s} \mathbb{R}^{m+1}\right)$ of smooth $s$-forms in $\Omega$ and the space $\mathcal{E}\left(\Omega ; \mathbb{R}_{0, m+1}^{(s)}\right)$ of smooth $\mathbb{R}_{0, m+1}^{(s)}$-valued functions in $\Omega$.

Indeed, associate with $\omega^{s}=\sum_{|A|=s} \omega_{A}^{s} d x^{A} \in \mathcal{E}\left(\Omega ; \wedge^{s} \mathbb{R}^{m+1}\right), \Theta \omega^{s}=W^{s}=\sum_{|A|=s} W_{A}^{s} e_{A} \in$ $\mathcal{E}\left(\Omega ; \mathbb{R}_{0, m+1}^{(s)}\right)$ where for all $A, W_{A}^{s}=\omega_{A}^{s}$.

Then clearly, through $\Theta$, the action of $\partial_{x}^{+}$and $\partial_{x}^{-}$on $\mathcal{E}\left(\Omega ; \mathbb{R}_{o, m+1}^{(s)}\right)$ as given in (1.10) corresponds, respectively, to the action of $d$ and $d^{*}$ on $\mathcal{E}\left(\Omega ; \wedge^{s} \mathbb{R}^{m+1}\right)$.

Notice in particular that on $\mathcal{E}\left(\Omega ; \mathbb{R}_{o, m+1}^{(s)}\right), \partial_{x}^{+2}=0$ and $\partial_{x}^{-2}=0$ whence, as $\partial_{x}^{2}=-\Delta_{x}, \Delta_{x}$ being the Laplacian in $\mathbb{R}^{m+1}$, we have that on $\mathcal{E}\left(\Omega ; \mathbb{R}_{o, m+1}^{(s)}\right), \partial_{x}^{+} \partial_{x}^{-}+\partial_{x}^{-} \partial_{x}^{+}=-\Delta_{x}$.

In [5] smooth differential forms $\omega$ satisfying $\left(d-d^{*}\right) \omega=0$ in $\Omega$ were called self-conjugate. It may be easily verified that if $W=\Theta \omega$, then

$$
\left(d-d^{*}\right) \omega=0 \Longleftrightarrow W \partial_{x}=0
$$

Now let $r \in\{0,1, \ldots, m+1\}$ be fixed and let $p, q \in N$ be such that $p<q$ and $r+2 q \leq m+1$. Then by $\mathbb{R}_{0, m+1}^{(r, p, q)}$ we denote the subspace of $\mathbb{R}_{0, m+1}$ defined by

$$
\mathbb{R}_{0, m+1}^{(r, p, q)}=\sum_{j=p}^{q} \oplus \mathbb{R}_{0, m+1}^{(r+2 j)} .
$$

For a $\mathbb{R}_{0, m+1}^{(r, p, q)}$-valued smooth function $W$ in $\Omega$ which decomposes as $W=\sum_{j=p}^{q} W^{r+2 j}$, we have (see also (1.11))

$$
\partial_{x} W=0 \Longleftrightarrow\left\{\begin{array}{l}
\partial_{x}^{-} W^{r+2 p}=0 \\
\partial_{x}^{+} W^{r+2 j}+\partial_{x}^{-} W^{r+2(j+1)}=0, j=p, \ldots, q-1 \\
\partial_{x}^{+} W^{r+2 q}=0
\end{array}\right.
$$

The system (1.13) is called a generalized Moisil-Théodoresco system of type $(r, p, q)$ ((GMT)system of type $(r, p, q))$. It was introduced in [1] where some general properties of solutions to this 
system have been investigated.

Of course, one could as well have considered the so-called (GMT)-system of type $(r, p, q)$ adjoint to (1.13) which corresponds to the equation $W \partial_{x}=0$. By virtue of (1.12), putting $\omega=\Theta^{-1} W$ would thus imply that $\omega$ satisfies the equation $\left(d-d^{*}\right) \omega=0$.

In the case $\mathbb{R}^{3}$ we refer to [9] for the notions of the (MT)-system and its adjoint.

The aim of the underlying paper is to study the space $M T^{+}\left(m+1 ; k ; \mathbb{R}_{0, m+1}^{(r, p, q)}\right)$ of $\mathbb{R}_{0, m+1}^{(r, p, q)}$-valued homogeneous polynomial solutions $W_{k}$ of degree $k(k \geq 1)$ in $\mathbb{R}^{m+1}$ to the system (1.13).

The study of the space $M T^{+}\left(m+1 ; k ; \mathbb{R}_{0, m+1}^{(r, p, q)}\right)$ is motivated by the fact that if $W$ is a solution to (1.13) in $\Omega$, then it is real-analytic in $\Omega$. This implies that if e.g. $O \in \Omega$, then in some open ball $\stackrel{\circ}{B}(O, R) \subset \Omega, W$ admits a Taylor expansion

$$
W(x)=\sum_{k=0}^{\infty} \sum_{|\gamma|=k} \frac{1}{\gamma^{!}} x^{\gamma} \partial^{\gamma} W(0),
$$

where as usual, for $\gamma=\left(\gamma_{0}, \gamma_{1}, \ldots, \gamma_{m}\right) \in \mathbb{N}^{m+1},|\gamma|=\sum_{i=0}^{m} \gamma_{i}, \gamma !=\gamma_{0} ! \gamma_{1} ! \ldots \gamma_{m} !, x^{\gamma}=x_{0}^{\gamma_{0}} x_{1}^{\gamma_{1}} \ldots x_{m}^{\gamma_{m}}$ and $\partial^{\gamma}=\partial_{x_{0}}^{\gamma_{0}} \partial_{x_{1}}^{\gamma_{1}} \ldots \partial_{x_{m}}^{\gamma_{m}}$.

Consequently, for each $k \in \mathbb{N}, k \geq 1$,

$$
W_{k}(x)=\sum_{|\gamma|=k} \frac{1}{\gamma !} x^{\gamma} \partial^{\gamma} W(0)
$$

is an element of $M T^{+}\left(m+1 ; k ; \mathbb{R}_{0, m+1}^{(r, p, q)}\right)$.

The following results are obtained:

(i) Let $\mathbb{R}_{0, m}$ be the Clifford algebra constructed over $\mathbb{R}^{0, m}$, the latter being the subspace of $\mathbb{R}^{0, m+1}$ spanned by $\underline{e}=\left(e_{1}, e_{2}, \ldots, e_{m}\right)$ and let $\mathbb{R}_{0, m+1}^{+}$and $\mathbb{R}_{0, m}^{+}$be the even subalgebras of $\mathbb{R}_{0, m+1}$ and $\mathbb{R}_{0, m}($ see $\S 2)$.

Then the operator $\overline{D_{x}}: \mathcal{H}\left(m+1 ; k+1 ; \mathbb{R}_{0, m}^{+}\right) \rightarrow M T^{+}\left(m+1 ; k ; \mathbb{R}_{0, m+1}^{+}\right)$is surjective (Corollary 4.3). The operator $\overline{D_{x}}$ is the conjugate of the Cauchy-Riemann operator $D_{x}=\bar{e}_{0} \partial_{x}$ in $\mathbb{R}^{m+1}$ where $\bar{e}_{0}=-e_{0}$ and $\mathcal{H}\left(m+1 ; k+1 ; R_{0, m}^{+}\right)$is the space of $\mathbb{R}_{0, m}^{+}$-valued solid harmonics of degree $k+1$ in $\mathbb{R}^{m+1}$.

Notice that in the case $m=1, \mathbb{R}_{0,2}^{+}=\mathbb{R} \oplus \bar{e}_{0} e_{1} \mathbb{R} \cong \mathbb{C}$ (identify $\bar{e}_{0} e_{1}$ with the imaginary unit), $\mathbb{R}_{0,1}^{+} \cong \mathbb{R}$ and that $\frac{1}{2} \bar{D}_{x}=\frac{1}{2}\left(\partial_{x_{0}}-\bar{e}_{0} e_{1} \partial_{x_{1}}\right)$ is nothing else but the operator $\partial_{z}$ in $\mathbb{R}^{2}$.

Corollary 4.3 thus generalizes to $\mathbb{R}^{m+1}$ the classical result in complex analysis stating that each homogeneous holomorphic polynomial of degree $k$ is the derivative (w.r.t. $\partial_{z}$ ) of a real-valued homogeneous harmonic polynomial of degree $k+1$.

Corollary 4.3 in fact follows from a more general result (Theorem 4.2), the proof of which relies heavily on the existence of conjugate harmonic pairs $\left(U_{k}, V_{k}\right)$ as treated in section 4.1 and on a refined version of the Poincaré Lemmas for the case of $r$-forms with homogeneous polynomial coefficients (see section $3)$. 
(ii) In the case $\mathbb{R}^{4}$, bases are constructed for the spaces $M T^{+}\left(4 ; k ; \mathbb{R}_{0,4}^{(r, p, q)}\right), r$ being even (see section $5)$. The fact that in studying (GMT)-systems of type $(r, p, q)$, we may restrict ourselves to the case $r$ even has been shown in section 2 .

(iii) It is proved in section 4.3 that each $W_{k} \in M T^{+}\left(m+1 ; k ; \mathbb{R}_{0, m+1}^{(r, p, q)}\right)$ admits a primitive $W_{k+1} \in$ $M T^{+}\left(m+1 ; k+1 ; \mathbb{R}_{0, m+1}^{(r, p, q)}\right)$, i.e. $W_{k}=\bar{D}_{x} W_{k+1}$.

\section{2 (GMT)-systems revisited}

Let again $\mathbb{R}_{0, m+1}$ be the Clifford algebra constructed over the quadratic space $\mathbb{R}^{0, m+1}$ with orthogonal basis $e=\left(e_{0}, e_{1}, \ldots, e_{m}\right)$ and let $\left(e_{A}:|A|=s, s=0,1, \ldots, m+1\right)$ be the standard basis of $\mathbb{R}_{0, m+1}$.

In (1.8), a decomposition of $\mathbb{R}_{0, m+1}$ in terms of its $r$-vector subspaces $\mathbb{R}_{0, m+1}^{(r)}, r=0,1, \ldots, m+1$, was obtained:

$$
\mathbb{R}_{0, m+1}=\sum_{r=0}^{m+1} \oplus \mathbb{R}_{0, m+1}^{(r)}
$$

The even subalgebra $\mathbb{R}_{0, m+1}^{+}$and the odd subspace $\mathbb{R}_{0, m+1}^{-}$of $\mathbb{R}_{0, m+1}$ are defined by

$$
\mathbb{R}_{0, m+1}^{+}=\sum_{r_{\mathrm{even}}} \oplus \mathbb{R}_{0, m+1}^{(r)}
$$

and

$$
\mathbb{R}_{0, m+1}^{-}=\sum_{r_{\text {odd }}} \oplus \mathbb{R}_{0, m+1}^{(r)}
$$

Obviously

$$
\mathbb{R}_{0, m+1}=\mathbb{R}_{0, m+1}^{+} \oplus \mathbb{R}_{0, m+1}^{-}
$$

The conjugation a $\rightarrow \bar{a}$ on $\mathbb{R}_{0, m+1}$ is defined by the basic properties

$$
\bar{e}_{i}=-e_{i}, i=0,1, \ldots, m
$$

and

$$
\overline{a b}=\bar{b} \quad \bar{a}, a, b \in \mathbb{R}_{0, m+1}
$$

The factorization $\mathbb{R}^{m+1}=\mathbb{R} \times \mathbb{R}^{m}$ leads to the following third decomposition of $\mathbb{R}_{0, m+1}$. Restrict the quadratic form on $\mathbb{R}^{m+1}$ to $\mathbb{R}^{m}$, thus obtaining the quadratic vector space $\mathbb{R}^{0, m}$ with orthogonal basis $\underline{e}=\left(e_{1}, \ldots, e_{m}\right)$.

Then inside $\mathbb{R}_{0, m+1}, \mathbb{R}^{0, m}$ generates the Clifford algebra $\mathbb{R}_{0, m}$ and clearly 


$$
\mathbb{R}_{0, m+1}=\mathbb{R}_{0, m} \oplus \bar{e}_{0} \mathbb{R}_{0, m}
$$

It thus follows that

$$
\mathbb{R}_{0, m+1}^{+}=\mathbb{R}_{0, m}^{+} \oplus \bar{e}_{0} \mathbb{R}_{0, m}^{-}
$$

and

$$
\mathbb{R}_{0, m+1}^{-}=\mathbb{R}_{0, m}^{-} \oplus \bar{e}_{0} \mathbb{R}_{0, m}^{+}
$$

Put $M=\{0,1, \ldots, m\}$ and $\stackrel{\circ}{M}=\{1, \ldots, m\}$ and consider the so-called pseudo-scalars $e_{M}=e_{0} e_{1} \ldots e_{m}$ and $e_{\stackrel{\circ}{\circ}}=e_{1} e_{2} \ldots e_{m}$ in, respectively, $\mathbb{R}_{0, m+1}$ and $\mathbb{R}_{0, m}$.

If $m+1$ is odd, then clearly right multiplication by $e_{M}$ determines an isomorphism $a^{-} \rightarrow a^{-} e_{M}, a^{-} \in$ $\mathbb{R}_{0, m+1}^{-}$, between $\mathbb{R}_{0, m+1}^{-}$and $\mathbb{R}_{0, m+1}^{+}$. In the case $m+1$ even, right multiplication by $e_{M}^{\circ}$ determines an isomorphism $a^{-} \rightarrow a^{-} e_{\dot{M}}$ between $\mathbb{R}_{0, m+1}^{-}$and $\mathbb{R}_{0, m+1}^{+}$. Indeed, by virtue of the decomposition (2.4), we have that, if $a^{-} \in \mathbb{R}_{0, m+1}^{-}$is written as

$$
a^{-}=b^{-}+\bar{e}_{0} b^{+}, b^{-} \in \mathbb{R}_{0, m}^{-}, b^{+} \in \mathbb{R}_{0, m}^{+},
$$

then $a^{-} e_{\stackrel{\circ}{M}} \in \mathbb{R}_{0, m+1}^{+}$with $a^{-} e_{\stackrel{\circ}{\circ}}=b^{-} e_{\stackrel{\circ}{\circ}}+\bar{e}_{0} b^{+} e_{\stackrel{\circ}{\circ}}$ where $b^{-} e_{\stackrel{\circ}{\circ}} \in \mathbb{R}_{0, m}^{+}$and $b^{+} e_{\stackrel{\circ}{M}} \in \mathbb{R}_{0, m}^{-}$. Now let $\Omega \subset \mathbb{R}^{m+1}$ be open and let $W: \Omega \rightarrow \mathbb{R}_{0, m+1}$ be a $C_{1}$-function in $\Omega$. Then $W$ is said to be left monogenic in $\Omega$ if $\partial_{x} W=0$ in $\Omega$ where $\partial_{x}=\sum_{i=0}^{m} e_{i} \partial_{x_{i}}$ is the Dirac operator in $\mathbb{R}^{m+1}$. By virtue of (2.2), $W$ splits into

$$
W=W^{+}+W^{-}
$$

where $W^{+}$and $W^{-}$are $R_{0, m+1^{-}}^{+}$and $\mathbb{R}_{0, m+1}^{-}$-valued $C_{1}$-functions in $\Omega$ Consequently

$$
\partial_{x} W=0 \Longleftrightarrow\left\{\begin{array}{l}
\partial_{x} W^{+}=0 \\
\partial_{x} W^{-}=0
\end{array}\right.
$$

If $\partial_{x} W=0$, then the set of components of $W^{+}$or of $W^{-}$was called in [18] a system of conjugate harmonic functions in $\Omega$.

Taking into account the isomorphisms $\mathbb{R}_{0, m+1}^{-} \rightarrow \mathbb{R}_{0, m+1}^{+}$where $a^{-} \rightarrow a^{-} e_{M}$ if $m+1$ is odd and $a^{-} \rightarrow a^{-} e_{\stackrel{\circ}{\circ}}$ if $m+1$ is even, $a^{-} \in \mathbb{R}_{0, m+1}^{-}$, it follows from (2.5) that it suffices in fact to study left monogenic $\mathbb{R}_{0, m+1}^{+}$-valued functions in $\Omega$.

Now let $W=\sum_{j=p}^{q} W^{r+2 j}$ be a $\mathbb{R}_{0, m+1}^{(r, p, q)}$-valued left monogenic function defined in $\Omega$, i.e. $W$ satisfies the so-called generalized Moisil-Théodoresco system of type $(r, p, q)$ (see also (1.13)).

$$
\partial_{x} W=0 \Longleftrightarrow\left\{\begin{array}{l}
\partial^{-} W^{r+2 p}=0 \\
\partial_{x}^{+} W^{r+2 j}+\partial_{x}^{-} W^{r+2(j H)}=0, j=p, \ldots, q-1 \\
\partial^{+} W^{r+2 q}=0
\end{array}\right.
$$


then in view of the observations made, it suffices to consider functions $W=\sum_{j=p}^{q} W^{r+2 j}$ where $r$ is even.

However, let us point out that if $W^{s}$ is $\mathbb{R}_{0, m+1}^{(s)}$-valued with $s$ odd then

(i) for $m+1$ odd, $W^{s} e_{M}$ is $\mathbb{R}_{0, m+1}^{(m+1-s)}$-valued while

(ii) for $m+1$ even, $W^{s} e_{\stackrel{\circ}{\circ}}$ is $\mathbb{R}_{0, m+1}^{(m-s)} \oplus \mathbb{R}_{0, m+1}^{(m-s+2)}$-valued

Property (i) being obvious, let us prove (ii).

Write out $W^{s}$ as $W^{s}=\sum_{|A|=s} W_{A}^{s} e_{A}$ and put for $A \subset\{0,1, \ldots, m\}$ with $|A|=s, A=\left\{i_{1}, i_{2}, \ldots, i_{s}\right\}$.

If $i_{1}=0$, then $A=e_{0} e_{B}$ where $B=\left\{i_{2}, \ldots, i_{s}\right\} \subset\{1, \ldots, m\}$ and so $e_{A} e_{M}^{\circ}=e_{0} e_{B} e_{M}^{\circ}= \pm e_{0} e_{M \backslash B}^{\circ} \in$ $\mathbb{R}_{0, m+1}^{(m-s+2)}$.

If $i_{1} \neq 0$, then $\left\{i_{1}, \ldots, i_{s}\right\} \subset\{1, \ldots, m\}$ and $e_{A} e_{M}^{\circ}= \pm e_{M \backslash A} \in \mathbb{R}_{0, m}^{(m-s)} \subset \mathbb{R}_{0, m+1}^{(m-s)}$.

We may thus conclude that for $s$ odd and $W \mathbb{R}_{0, m+1}^{(s, p, q)}$-valued in $\Omega$, in the case $(m+1)$ odd, $W e_{M}$ is $\mathbb{R}_{0, m+1}^{\left(s^{*}, 0, q^{*}\right)}$-valued where $s^{*}=m+1-(s+2 q)$ and $q^{*}=q-p$, while in the case $(m+1)$ even, $W e_{M}$ is $\mathbb{R}_{0, m+1}^{\left(s^{*}, 0, q^{*}\right)}$-valued where $s^{*}=m-s-2 q$ and $q^{*}=q-p+1$.

These properties are nicely illustrated in the cases $\mathbb{R}^{3}$ and $\mathbb{R}^{4}$.

In $\mathbb{R}^{3}$, the following $(r, p, q)$-subspaces occur:

(i) for $r$ even: $\mathbb{R}_{0,3}^{(0)} \oplus \mathbb{R}_{0,3}^{(2)}=\mathbb{R}_{0,3}^{+}$

(ii) for $r$ odd: $\mathbb{R}_{0,3}^{(1)} \oplus \mathbb{R}_{0,3}^{(3)}=\mathbb{R}_{0,3}^{-}$

and

$$
\mathbb{R}_{0,3}^{-} e_{M}=\mathbb{R}_{0,3}^{-} e_{0} e_{1} e_{2}=\mathbb{R}_{0,3}^{+} .
$$

In $\mathbb{R}^{4}$, we have:

(i) for $r$ even:

$$
\begin{gathered}
\mathbb{R}_{0,4}^{(0,0,1)}=\mathbb{R}_{0,4}^{(0)} \oplus \mathbb{R}_{0,4}^{(2)} ; \quad \mathbb{R}_{0,4}^{(2,0,1)}=\mathbb{R}_{0,4}^{(2)} \oplus \mathbb{R}_{0,4}^{(4)} \\
\mathbb{R}_{0,4}^{(0,0,2)}=\mathbb{R}_{0,4}^{(0)} \oplus \mathbb{R}_{0,4}^{(2)} \oplus \mathbb{R}_{0,4}^{(4)}=\mathbb{R}_{0,4}^{+}
\end{gathered}
$$

(ii) for $r$ odd:

$$
\mathbb{R}_{0,4}^{(1,0,1)}=\mathbb{R}_{0,4}^{(1)} \oplus \mathbb{R}_{0,4}^{(3)}=\mathbb{R}_{0,4}^{-}
$$

and obviously

$$
\mathbb{R}_{0,4}^{-} e_{M}^{\circ}=\mathbb{R}_{0,4}^{-} e_{1} e_{2} e_{3}=\mathbb{R}_{0,4}^{(0,0,2)}=\mathbb{R}_{0,4}^{+} .
$$

\section{The Poincaré Lemmas revisited}

The Poincaré Lemmas on closed and co-closed differential forms in an open subset $\Omega$ of $\mathbb{R}^{m+1}$ are well-known. To our knowledge, their refined versions are less known in classical literature and so are their statements in terms of homogeneous polynomials.

As in characterizing homogeneous polynomial solutions of (GMT)-systems of type $(r, p, q)$, these versions of the Poincaré Lemmas and some of their applications will play a central role (see $\S 4$ ), for convenience of the reader we restate them in full detail. Most of the proofs will be omitted since they 
may be given by following classical lines of reasoning, as e.g. worked out in [3].

Throughout this section, the following notations will be kept on. For $r, k \in \mathbb{N}$ with $0 \leq r \leq m+1$ and $k \geq 1, \Phi_{k}^{r}$ and $\mathcal{P}_{k}^{r}$ denote the spaces of $\wedge^{r} \mathbb{R}^{m+1}$-valued and $\mathbb{R}_{0, m+1}^{(r)}$-valued homogeneous polynomials of degree $k$ in $\mathbb{R}^{m+1}$, i.e. $\Phi_{k}^{r}=$

$\mathcal{P}(m+1 ; k) \otimes \mathbb{R} \wedge^{r} \mathbb{R}^{m+1}$ and $\mathcal{P}_{k}^{r}=\mathcal{P}(m+1 ; k) \otimes \mathbb{R} \mathbb{R}_{0, m+1}^{(r)}$, where $\mathcal{P}(m+1 ; k)$ is the space of homogeneous real-valued homogeneous polynomials of degree $k$ in $\mathbb{R}^{m+1}$.

Arbitrary elements of $\Phi_{k}^{r}$ and $\mathcal{P}_{k}^{r}$ will be denoted by $\omega_{k}^{r}$ and $P_{k}^{r}$.

Furthermore, the spaces $k e r_{k}^{r} \partial_{x}^{+}$and $\operatorname{ker}_{k}^{r} \partial_{x}^{-}$are defined by

$$
\operatorname{ker}_{k}^{r} \partial_{x}^{+}=\left\{P_{k}^{r} \in \mathcal{P}_{k}^{r}: \partial_{x}^{+} P_{k}^{r}=0\right\}
$$

and

$$
\operatorname{ker}_{k}^{r} \partial_{x}^{-}=\left\{P_{k}^{r} \in \mathcal{P}_{k}^{r}: \partial_{x}^{-} P_{k}^{r}=0\right\}
$$

\subsection{The classical Poincaré Lemmas on $\mathcal{P}_{k}^{r}$}

In [3] it was shown that for each $\omega_{k}^{r} \in \Phi_{k}^{r}$,

$$
(E\rfloor d+d E\rfloor) \omega_{k}^{r}=(k+r) \omega_{k}^{r}
$$

where the operator $E\rfloor$ is defined by

$$
\left.E\rfloor=\sum_{j=0}^{m} x_{j} \partial_{x_{j}}\right\rfloor
$$

$\left.\partial_{x_{j}}\right\rfloor$ being the contraction operator acting on a basic $r$-differential form $d x^{A}=d x^{i_{1}} \wedge d x^{i_{2}} \wedge \ldots \wedge$ $d x^{i_{r}}$, i.e.

$$
\left.\partial_{x_{j}}\right\rfloor d x^{A}=\sum_{l=1}^{r}(-1)^{l-1} \delta_{j i_{l}} d x^{A \backslash\left\{i_{l}\right\}}
$$

¿From (3.1) it thus follows that if $d \omega_{k}^{r}=0$ with $\omega_{k}^{r}=\sum_{|A|=r} w_{k, A}^{r} d x^{A}$, then the element $\omega_{k+1}^{r-1} \in$ $\Phi_{k+1}^{r-1}$ given by

$$
\left.\omega_{k+1}^{r-1}=\frac{1}{k+r} E\right\rfloor \omega_{k}^{r}=\frac{1}{k+r} \sum_{|A|=r} \omega_{k, A}^{r}\left(\sum_{j=1}^{r}(-1)^{j-1} x_{i_{j}} d x^{A \backslash\left\{i_{j}\right\}}\right)
$$

is such that

$$
\omega_{k}^{r}=d \omega_{k+1}^{r-1}
$$

Through the isomophism $\Theta$ (see $\S 1$ ) we have thus proved.

Lemma 3.1 (Poincaré Lemma) Let $r \geq 1$ and let $k \in \mathbb{N}$. Then for $P_{k}^{r} \in \mathcal{P}_{k}^{r}$ the following properties 
are equivalent:

(i) $\partial_{x}^{+} P_{k}^{r}=0$

(ii) there exists $P_{k+1}^{r-1} \in \mathcal{P}_{k+1}^{r-1}$ such that $P_{k}^{r}=\partial_{x}^{+} P_{k+1}^{r-1}$.

It is clear that by right multiplication with the pseudo-scalar $e_{M}$ an isomorphism is obtained between the spaces $\mathcal{P}_{k}^{r}$ and $\mathcal{P}_{k}^{m+1-r}$, its inverse being given by right multiplication with $\epsilon_{M} e_{M}$, where $\epsilon_{M}=e_{M}^{2}= \pm 1$. As furthermore for each $P_{k}^{r} \in \mathcal{P}_{k}^{r}, \partial_{x}\left(P_{k}^{r} e_{M}\right)=\left(\partial_{x} P_{k}^{r}\right) e_{M}$, we have that

$$
\left(\partial_{x}^{+} P_{k}^{r}\right) e_{M}=\partial_{x}^{-}\left(P_{k}^{r} e_{M}\right)
$$

and

$$
\left(\partial_{x}^{-} P_{k}^{r}\right) e_{M}=\partial_{x}^{+}\left(P_{k}^{r} e_{M}\right)
$$

Combining the relation (3.2) with Lemma 3.1 yields

Lemma 3.2 (Dual Poincaré Lemma) Let $r<m+1$ and let $k \in \mathbb{N}$. Then for $P_{k}^{r} \in \mathcal{P}_{k}^{r}$ the following properties are equivalent:

(i) $\partial_{x}^{-} P_{k}^{r}=0$

(ii) there exists $P_{k+1}^{r+1} \in \mathcal{P}_{k+1}^{r+1}$ such that $P_{k}^{r}=\partial_{x}^{-} P_{k+1}^{r+1}$.

Refined versions of the Poincaré Lemmas may now be easily deduced.

\section{Lemma 3.3}

(i) Let $r \geq 1$ and let $P_{k}^{r} \in \mathcal{P}_{k}^{r}$. Then the following properties are equivalent:

(i.1) $\partial_{x}^{+} P_{k}^{r}=0$

(i.2) there exists $P_{k+1}^{r-1} \in \mathcal{P}_{k+1}^{r-1}$ such that $\partial_{x}^{-} P_{k+1}^{r-1}=0$ and $P_{k}^{r}=\partial_{x}^{+} P_{k+1}^{r-1}$.

(ii) Let $r<m+1$ and let $P_{k}^{r} \in \mathcal{P}_{k}^{r}$. Then the following properties are equivalent:

(ii.1) $\partial_{x}^{-} P_{k}^{r}=0$

(ii.2) there exists $P_{k+1}^{r+1} \in \mathcal{P}_{k+1}^{r+1}$ such that $\partial_{x}^{+} P_{k+1}^{r+1}=0$ and $P_{k}^{r}=\partial_{x}^{-} P_{k+1}^{r+1}$.

\subsection{The surjectivity of the operators $\partial_{x}^{-} \partial_{x}^{+}$and $\partial_{x}^{+} \partial_{x}^{-}$}

Let $P_{k}^{r} \in k e r_{k}^{r} \partial_{x}^{+}$, i.e. $\partial_{x}^{+} P_{k}^{r}=0$.

By means of Lemma 3.1, there exists $P_{k+1}^{r-1} \in \mathcal{P}_{k+1}^{r-1}$ such that $P_{k}^{r}=\partial_{x}^{+} P_{k+1}^{r-1}$.

As $\Delta_{x}: \mathcal{P}_{k+3}^{r-1} \rightarrow \mathcal{P}_{k+1}^{r-1}$ is surjective (see e.g. [10]) there exists $P_{k+3}^{r-1} \in \mathcal{P}_{k+3}^{r-1}$ such that $P_{k+1}^{r-1}=$ $-\Delta_{x} P_{k+3}^{r-1}$.

Put $P_{k+2}^{r}=\partial_{x}^{+} P_{k+3}^{r-1}$. Then $\partial_{x}^{+} P_{k+2}^{r}=0$.

Moreover, on the one hand 


$$
\begin{aligned}
-\Delta_{x} P_{k+2}^{r} & =\left(\partial_{x}^{+} \partial_{x}^{-}+\partial_{x}^{-} \partial_{x}^{+}\right)\left(\partial_{x}^{+} P_{k+3}^{r-1}\right) \\
& =\partial_{x}^{+} \partial_{x}^{-} P_{k+2}^{r}
\end{aligned}
$$

while on the other hand

$$
\begin{aligned}
-\Delta_{x} P_{k+2}^{r} & =-\Delta_{x}\left(\partial_{x}^{+} P_{k+3}^{r-1}\right) \\
& =\partial_{x}^{+}\left(-\Delta_{x} P_{k+3}^{r-1}\right) \\
& =\partial_{x}^{+} P_{k+1}^{r-1} \\
& =P_{k}^{r} .
\end{aligned}
$$

It thus follows that, given $P_{k}^{r} \in k e r_{k}^{r} \partial_{x}^{+}$, there exists $P_{k+2}^{r} \in k e r_{k}^{r} \partial_{x}^{+}$such that $P_{k}^{r}=\partial_{x}^{+} \partial_{x}^{-} P_{k+2}^{r}$. In a similar way, it may be proved that, given $P_{k}^{r} \in k e r_{k}^{r} \partial_{x}^{-}$, there exists $P_{k+2}^{r} \in k e r_{k+2}^{r} \partial_{x}^{-}$such that $P_{k}^{r}=\partial_{x}^{-} \partial_{x}^{+} P_{k+2}^{r}$.

We thus obtain

Lemma 3.4 The differential operators

(i) $\partial_{x}^{-} \partial_{x}^{+}: \operatorname{ker}_{k+2}^{r} \partial_{x}^{-} \rightarrow \operatorname{ker}_{k}^{r} \partial_{x}^{-}$

and

(ii) $\partial_{x}^{+} \partial_{x}^{-}: \operatorname{ker}_{k+2}^{r} \partial_{x}^{+} \rightarrow \operatorname{ker}_{k}^{r} \partial_{x}^{+}$

are surjective.

\section{Conjugate harmonicity-Harmonic potentials-Primitives}

Let the $\mathbb{R}_{0, m+1}$-valued $C_{1}$-function $F$ defined in some open domain $\Omega \subset \mathbb{R}^{m+1}$ be decomposed following (2.3), i.e.

$$
F=U+\bar{e}_{0} V
$$

where $U$ and $V$ are $\mathbb{R}_{0, m}$-valued $C_{1}$-functions in $\Omega$.

Furthermore, let $\partial_{\underline{x}}=\sum_{j=1}^{m} e_{j} \partial_{x_{j}}$ be the Dirac operator in $\mathbb{R}^{m}$ and let $D_{x}=\bar{e}_{0} \partial_{x}=\partial_{x_{0}}+\bar{e}_{0} \partial_{\underline{x}}$ be the Cauchy-Riemann operator in $\mathbb{R}^{m+1}$.

Notice that the conjugate $\bar{D}_{x}$ of $D_{x}$ is given by $\bar{D}_{x}=\partial_{x_{0}}-\bar{e}_{0} \partial_{\underline{x}}$ and that $\bar{D}_{x} D_{x}=D_{x} \bar{D}_{x}=\Delta_{x}$. Then the following properties are equivalent in $\Omega$ :

$$
\partial_{x} F=0 \Leftrightarrow D_{x} F=0 \Leftrightarrow\left\{\begin{array}{l}
\partial_{x_{0}} U+\partial_{\underline{x}} V=0 \\
\partial_{\underline{x}} U+\partial_{x_{0}} V=0
\end{array}\right.
$$


The system (4.2) clearly generalizes the classical Cauchy-Riemann system in the plane. As left monogenic functions are real-analytic and $\partial_{x}^{2}=-\Delta_{x}$, it follows that a pair $(U, V)$ of $\mathbb{R}_{0, m^{-}}$ valued $C_{1}$-functions in $\Omega$ satisfying (4.2) is automatically a pair of $\mathbb{R}_{0, m}$-valued harmonic functions in $\Omega$. Such a pair $(U, V)$ is called a conjugate harmonic pair in $\Omega$.

\subsection{Conjugate harmonicity}

Let the integers $r, p, q$ be as in section 2 with $r$ even.

In this section we solve the following problem:

(P1) Given $U_{k}=\sum_{j=p}^{q} U_{k}^{r+2 j}$, a $\mathbb{R}_{0, m}^{(r, p, q)}$-valued homogeneous harmonic polynomial of degree $k$ in $\mathbb{R}^{m+1}$, under which conditions does there exist a $\mathbb{R}_{0, m}^{(r-1, p, q)}$-valued homogeneous harmonic polynomial $\widetilde{V}_{k}$ of degree $k$ in $\mathbb{R}^{m+1}$ such that $\left(U_{k}, \widetilde{V}_{k}\right)$ is a conjugate harmonic pair, i.e. such that

$$
\widetilde{W}_{k}=U_{k}+\bar{e}_{0} \widetilde{V}_{k} \in M T^{+}\left(m+1 ; k ; \mathbb{R}_{o, m+1}^{(r, p, q)}\right) .
$$

In what follows we denote by $\mathcal{H}\left(m+1 ; k ; \mathbb{R}_{0, m}^{(r, p, q)}\right)$ the space of $\mathbb{R}_{0, m}^{(r, p, q)}$-valued homogeneous harmonic polynomials of degree $k$ in $\mathbb{R}^{m+1}$, i.e. $\mathcal{H}\left(m+1 ; k ; \mathbb{R}_{0, m}^{(r, p, q)}\right)=\mathcal{H}(m+1 ; k) \otimes_{\mathbb{R}} \mathbb{R}_{0, m}^{(r, p, q)}$, where $\mathcal{H}(m+1 ; k)$ is the space of solid harmonics of degree $k$ in $\mathbb{R}^{m+1}$.

Putting $\widetilde{V}_{k}=\sum_{j=p}^{q} \widetilde{V}_{k}^{r-1+2 j}$, the condition (4.3), or equivalently the condition (4.2), leads to the following systems to be satisfied

$$
\left\{\begin{array}{l}
\partial_{\underline{x}}^{-} \widetilde{V}_{k}^{r-1+2 p}=0 \\
\partial_{x_{0}} U_{k}^{r+2 j}+\partial_{\underline{x}}^{+} \widetilde{V}_{k}^{r-1+2 j}+\partial_{\underline{x}}^{-} \widetilde{V}_{k}^{r-1+2 j+2}=0, j=p, \ldots, q-1 \\
\partial_{x_{0}} U_{k}^{r+2 q}+\partial_{\underline{x}}^{+} \widetilde{V}_{k}^{r-1+2 q}=0
\end{array}\right.
$$

and

$$
\left\{\begin{array}{l}
\partial_{\underline{x}}^{-} U_{k}^{r+2 p}+\partial_{x_{0}} \widetilde{V}_{k}^{r-1+2 p}=0 \\
\partial_{\underline{x}}^{+} U_{k}^{r+2 j}+\partial_{\underline{x}}^{-} U_{k}^{r+2 j+2}+\partial_{x_{0}} \widetilde{V}_{k}^{r-1+2 j+2}=0 \\
\partial_{\underline{x}}^{+} U_{k}^{r+2 q}=0
\end{array}\right.
$$

¿From (4.5), it thus follows that a necessary condition on $U_{k}$ to be fulfilled is that $\partial_{\widetilde{\underline{V}}}^{+} U_{k}^{r+2 q}=0$. We now claim that the latter condition is also sufficient to ensure the existence of a $\widetilde{V}_{k}$ conjugate harmonic to $U_{k}$, i.e. $\widetilde{W}_{k}=U_{k}+\bar{e}_{0} \widetilde{V}_{k} \in M T^{+}\left(m+1 ; k ; \mathbb{R}_{0, m+1}^{(r, p, q)}\right)$.

The proof may be given along the same lines worked out in [2] for the construction of conjugate harmonic pairs in general, on the understanding that, where necessary, the arguments used should be adapted to the case of homogeneous polynomials.

That is why we do not work it out in full detail.

A first step consists in constructing

$$
\widetilde{H}_{k+1}(x)=\int_{0}^{x_{0}} U_{k}(t, \underline{x}) d t-\widetilde{h}_{k+1}(\underline{x})
$$


where $\widetilde{h}_{k+1} \in \mathcal{P}(m ; k+1) \otimes \mathbb{R} \mathbb{R}_{0, m}^{(r, p, q)}$ satisfies the equation

$$
\Delta_{\underline{x}} \widetilde{h}_{k+1}(\underline{x})=\partial_{x_{0}} U_{k}(0, \underline{x}) .
$$

As $\Delta_{\underline{x}}: \mathcal{P}(m ; k+1) \rightarrow \mathcal{P}(m ; k-1)$ is surjective, such $\widetilde{h}_{k+1}$ does exist. Even more, due to the Fischer decomposition (see [10]) the equation (4.7) admits a unique solution of the form

$$
\widetilde{h}_{k+1}(\underline{x})=|\underline{x}|^{2} s_{k-1}(\underline{x})
$$

where $s_{k-1} \in \mathcal{P}(m ; k-1) \otimes \mathbb{R} \mathbb{R}_{0, m}^{(r, p, q)}$.

It may be easily verified that $\widetilde{H}_{k+1} \in \mathcal{H}\left(m+1 ; k+1 ; \mathbb{R}_{0, m}^{(r ; p ; q)}\right)$.

Fix such a solution to (4.7) and write it out as

$$
\widetilde{h}_{k+1}(\underline{x})=\sum_{j=p}^{q} \widetilde{h}_{k+1}^{r+2 j}(\underline{x}) .
$$

A second step consists in proving the existence of $h_{k+1}^{r+2 q} \in \mathcal{P}(m ; k+1) \otimes \mathbb{R}^{\mathbb{R}_{0, m}^{(r+2 q)}}$ such that

$$
W_{k+1}^{r+2 q}(\underline{x})=\widetilde{h}_{k+1}^{r+2 q}+h_{k+1}^{r+2 q}(\underline{x})
$$

satisfies the equations

$$
\left\{\begin{array}{l}
\partial_{\underline{x}}^{+} W_{k+1}^{r+2 q}=0 \\
\partial_{\underline{x}}^{+} \partial_{\underline{x}}^{-} W_{k+1}^{r+2 q}=-\partial_{x_{0}} U_{k}^{r+2 q}(0, \underline{x})
\end{array}\right.
$$

To this end first notice that, as $\partial_{\underline{x}}^{+} U_{k}^{r+2 q}=0$, we have that $\partial_{x_{0}} U_{k}^{r+2 q}(0, \underline{x}) \in k e r_{k-1}^{r+2 q} \partial_{\underline{x}}^{+}$. As $\partial_{\underline{x}}^{+} \partial_{\underline{x}}^{-}: k e r_{k+1}^{r+2 q} \partial_{\underline{x}}^{+} \rightarrow k e r_{k-1}^{r+2 q} \partial_{\underline{x}}^{+}$is surjective (see Lemma 3.4), $W_{k+1}^{r+2 q} \in k e r_{k+1}^{r+2 q} \partial_{\underline{x}}^{+}$satisfying (4.8) may be found.

Put $h_{k+1}^{r+2 q}=W_{k+1}^{r+2 q}-\widetilde{h}_{k+1}^{r+2 q}$. Then it may be easily checked that $h_{k+1}^{r+2 q} \in$ $\mathcal{H}(m ; k+1) \otimes_{\mathbb{R}} \mathbb{R}_{0, m}^{(r+2 q)}$.

By construction it thus follows that

$$
H_{k+1}^{*}(x)=\widetilde{H}_{k+1}(x)-h_{k+1}^{r+2 q}(\underline{x}) \in \mathcal{H}\left(m+1 ; k+1 ; \mathbb{R}_{0, m}^{(r, p, q)}\right)
$$

with

$$
\partial_{\underline{x}}^{+} H_{k+1}^{* r+2 q}=0 .
$$

Consequently $\widetilde{W}_{k}=\bar{D}_{x} H_{k+1}^{*}=\widetilde{U}_{k}+\bar{e}_{0} \widetilde{V}_{k} \in M T^{+}\left(k ; \mathbb{R}_{0, m+1}^{(r, p, q)}\right)$ with $\widetilde{U}_{k}=\partial_{x_{0}} H_{k+1}^{*}=U_{k}$ and $\widetilde{V}_{k}=-\partial_{\underline{x}} H_{k+1}^{*}$. 
Let $\mathcal{H}_{+}\left(m+1 ; k ; \mathbb{R}_{0, m}^{(r, p, q)}\right)$ denote the space of those elements $U_{k} \in \mathcal{H}\left(m+1 ; k ; \mathbb{R}_{0, m}^{(r, p, q)}\right)$ such that $\partial_{\underline{x}}^{+} U_{k}^{r+2 q}=0$. Then we have proved

Theorem 4.1. Let $U_{k} \in \mathcal{H}\left(m+1 ; k ; \mathbb{R}_{0, m}^{(r, p, q)}\right)$. Then $U_{k}$ admits a conjugate harmonic $\widetilde{V}_{k} \in$ $\mathcal{H}\left(m+1 ; k ; \mathbb{R}_{0, m}^{(r-1, p, q)}\right)$ if and only if $U_{k} \in \mathcal{H}_{+}\left(m+1 ; k ; \mathbb{R}_{0, m}^{(r, p, q)}\right)$.

Remarks(1) In constructing $\tilde{V}_{k}$ conjugate harmonic to $U_{k}$, the harmonic potential $H_{k+1}^{*}$ obtained belongs to $\mathcal{H}_{+}\left(m+1 ; k+1 ; \mathbb{R}_{0, m}^{(r, p, q)}\right)($ see $(4.9))$.

(2) Notice that the condition on $U_{k}$ in Theorem 4.1 is automatically satisfied when the (GMT)system of type $(r, p, q)$ considered, $r$ being even, is such that when $m+1$ is even, $r+2 q=m+1$ or when $m+1$ is odd, $r+2 q=m$.

Indeed, let $U_{k}=\sum_{j=p}^{q} U_{k}^{r+2 j} \in \mathcal{H}\left(m+1 ; k ; \mathbb{R}_{0, m}^{(r, p, q)}\right)$ be given.

If $m+1=r+2 q$, then clearly $U_{k}^{r+2 q}=U_{k}^{m+1}=0$ and this since $m<r+2 q=m+1$. Consequently the condition $\partial_{\underline{x}}^{+} U_{k}^{r+2 q}=\partial_{\underline{x}}^{+} U_{k}^{m+1}=0$ holds.

Moreover, as we then have that $\partial_{x_{0}} U_{k}^{m+1}=0$, a solution $\tilde{h}_{k+1}$ to the equation (4.6) may be chosen having its $\tilde{h}_{k+1}^{m+1}$-term identically zero. Finally, no correction term $h_{k+1}^{m+1}$ should then be taken.

If $m=r+2 q$, then $\partial_{\underline{x}}^{+} U_{k}^{r+2 q}=\left[\partial_{\underline{x}} U_{k}^{r+2 q}\right]_{m+1}=0$ and so again the condition $\partial_{\underline{x}}^{+} U_{k}^{r+2 q}=0$ is satisfied.

Notice that the foregoing situations are clearly met when $r=0, p=0$ and $q=\left[\frac{m+1}{2}\right]$, i.e. when $U_{k}$ is $\mathbb{R}_{0, m}^{+}$-valued.

Remark (2) implies

Proposition 4.2 (i) Let $r$ be even. If $m+1$ is even and $r+2 q=m+1$ or $m+1$ is odd and $r+2 q=m$, then

$$
\begin{aligned}
& \mathcal{H}_{+}\left(\mathbb{R}^{m+1} ; k ; \mathbb{R}_{0, m}^{(r, p, q)}\right)=\mathcal{H}\left(\mathbb{R}^{m+1} ; k ; \mathbb{R}_{0, m}^{(r, p, q)}\right) \\
& \text { (ii) } \mathcal{H}_{+}\left(\mathbb{R}^{m+1} ; k ; \mathbb{R}_{0, m}^{+}\right)=\mathcal{H}\left(\mathbb{R}^{m+1} ; k ; \mathbb{R}_{0, m}^{+}\right) .
\end{aligned}
$$

\subsection{Harmonic potentials}

Let again $r, p, q \in \mathbb{N}$ be as in section 2 and let $r$ be even.

In this section, we solve the following problem:

(P2) Let $W_{k} \in M T^{+}\left(m+1 ; k ; \mathbb{R}_{0, m+1}^{(r, p, q)}\right)$ be given. Find $H_{k+1} \in \mathcal{H}_{+}\left(m+1 ; k+1 ; \mathbb{R}_{0, m}^{(r, p, q)}\right)$ such that $W_{k}=\bar{D}_{x} H_{k+1}$. 
To this end we first prove

Lemma 4.3 Let $W_{k} \in \mathcal{P}(m+1 ; k) \otimes_{\mathbb{R}} \mathbb{R}_{0, m+1}^{(r, p, q)}$. Then the following properties are equivalent:

(i) $W_{k} \in M T^{+}\left(m+1 ; k ; \mathbb{R}_{0, m+1}^{(r, p, q)}\right)$

(ii) there exists $H_{k+1} \in \mathcal{H}(m+1 ; k+1) \otimes \mathbb{R} \mathbb{R}_{0, m+1}^{(r+1, p, q-1)}$ such that $W_{k}=\partial_{x} H_{k+1}$.

Proof. It is clear that if $H_{k+1} \in \mathcal{H}(m+1 ; k+1) \otimes \mathbb{R} \mathbb{R}_{0, m+1}^{(r+1, p, q-1)}$ then $W_{k}=\partial_{x} H_{k+1} \in$ $\mathcal{P}(m+1 ; k) \otimes \mathbb{R} \mathbb{R}_{0, m+1}^{(r, p, q)}$. As $\partial_{x}^{2}=-\Delta_{x}, W_{k} \in M T^{+}\left(m+1 ; k ; \mathbb{R}_{0, m+1}^{(r, p, q)}\right)$ and so (ii) $\rightarrow$ (i) is proved. Conversely, assume that $W_{k} \in M T^{+}\left(m+1 ; k ; \mathbb{R}_{0, m+1}^{(r, p, q)}\right)$ and put $W_{k}=\sum_{j=p}^{q} W_{k}^{r+2 j}$. As $\partial_{x} W_{k}=0$, the sequence $\left(W_{k}^{r+2 j}\right)_{j=p}^{q}$ satisfies the system (2.6), i.e.

$$
\left\{\begin{array}{l}
\partial_{x}^{-} W_{k}^{r+2 q}=0 \\
\partial_{x}^{+} W_{k}^{r+2 j}+\partial_{x}^{-} W_{k}^{r+2(j+1)}=0, j=p, \ldots, q-1 \\
\partial_{x}^{+} W_{k}^{r+2 q}=0
\end{array}\right.
$$

By virtue of Lemma 3.3, the first and last equation in (4.10) imply the existence of $W_{k+1}^{r+2 p+1} \in$ $\mathcal{P}_{k+1}^{r+2 p+1}$ and of $W_{k+1}^{r+2 q-1} \in \mathcal{P}_{k+1}^{r+2 q-1}$ such that $\partial_{x}^{+} W_{k+1}^{r+2 p+1}=0$ and $W_{k}^{r+2 p}=\partial_{x}^{-} W_{k+1}^{r+2 p+1}$, respectively, $\partial_{x}^{-} W_{k+1}^{r+2 q-1}=0$ and $W_{k}^{r+2 q}=\partial_{x}^{+} W_{k+1}^{r+2 q-1}$.

Put $W_{k}^{*}=\sum_{j=p+1}^{q-1} W_{k}^{r+2 j}$. Then $W_{k}^{*} \in \mathcal{P}(m+1 ; k) \otimes \mathbb{R} \mathbb{R}_{0, m+1}^{(r, p+1, q-1)}$.

As $\Delta_{x}: \mathcal{P}(m+1 ; k+2) \otimes \mathbb{R}_{\mathbb{R}} \mathbb{R}_{0, m+1}^{(r, p+1 ; q-1)} \rightarrow \mathcal{P}(m+1 ; k) \otimes \mathbb{R} \mathbb{R}_{0, m+1}^{(r, p+1, q-1)}$ is surjective, there ought to exist $W_{k+2}^{*} \in \mathcal{P}(m+1 ; k+2) \otimes \mathbb{R} \mathbb{R}_{0, m+1}^{(r, p+1, q-1)}$ such that $\Delta_{x} W_{k+2}^{*}=W_{k}^{*}$.

Putting

$$
H_{k+1}=W_{k+1}^{r+2 p+1}-\partial_{x} W_{k+2}^{*}+W_{k+1}^{r+2 q-1}
$$

it is clear that $H_{k+1} \in \mathcal{P}(m+1 ; k+1) \otimes \mathbb{R} \mathbb{R}_{0, m+1}^{(r+1, p, q-1)}$ and that $\partial_{x} H_{k+1}=W_{k}$.

Furthermore, as $\partial_{x}^{2}=-\Delta_{x}, \partial_{x} W_{k}=0$ implies that $W_{k+1}$ is harmonic, whence the implication (ii) $\rightarrow$ (i) is proved.

Now let $H_{k+1} \in \mathcal{H}_{+}\left(m+1 ; k+1 ; \mathbb{R}_{0, m}^{(r, p, q)}\right)$ be given. Then obviously $W_{k}=\bar{D}_{x} H_{k+1} \in M T^{+}(m+$ $\left.1 ; k ; \mathbb{R}_{0, m+1}^{(r, p, q)}\right)$. Conversely, assume that $W_{k} \in M T^{+}\left(m+1 ; k ; \mathbb{R}_{0, m+1}^{(r, p, q)}\right)$ and decompose $W_{k}$ following $(4.1)$, i.e.

$$
W_{k}=U_{k}+\bar{e}_{0} V_{k}
$$


where $U_{k} \in \mathcal{H}_{+}\left(m+1 ; k ; \mathbb{R}_{0, m}^{(r, p, q)}\right)$ and $V_{k} \in \mathcal{H}\left(m+1 ; k ; \mathbb{R}_{0, m}^{(r-1, p, q)}\right)$. Associate with $U_{k}$ the harmonic potential $H_{k+1}^{*} \in H_{+}\left(m+1 ; k+1 ; \mathbb{R}_{0, m}^{(r, p, q)}\right)$ obtained in (4.9), i.e. $\widetilde{W}_{k}=\widetilde{U}_{k}+\bar{e}_{0} \widetilde{V}_{k}=\bar{D}_{x} H_{k+1}^{*} \in M T^{+}\left(m+1 ; k ; \mathbb{R}_{0, m+1}^{(r, p, q)}\right)$ with $\widetilde{U}_{k}=U_{k}$ and $\widetilde{V}_{k}=-\partial_{\underline{x}} H_{k+1}^{*}$.

¿From $\widetilde{W}_{k}-W_{k}=\bar{e}_{0}\left(\widetilde{V}_{k}-V_{k}\right) \in M T^{+}\left(m+1 ; k ; \mathbb{R}_{0, m+1}^{(r, p, q)}\right)$, i.e. $D_{x}\left(\widetilde{W}_{k}-W_{k}\right)=0$, it easily follows from (4.2) that $\widetilde{V}_{k}-V_{k}$ is independent of $x_{0}$ and that $\widetilde{V}_{k}-V_{k} \in M T^{+}\left(m ; k ; \mathbb{R}_{0, m}^{(r-1, p, q)}\right)$. Put

By virtue of Lemma 4.3 , there exists $H_{k+1}^{* *} \in \mathcal{H}\left(m ; k+1, \mathbb{R}_{0, m}^{(r, p, q-1)}\right)$ such that $\widetilde{V}_{k}-V_{k}=\partial_{\underline{x}} H_{k+1}^{* *}$.

$$
L_{k+1}=H_{k+1}^{*}+H_{k+1}^{* *} .
$$

Then by construction $L_{k+1} \in \mathcal{H}_{+}\left(m+1 ; k+1 ; \mathbb{R}_{0, m}^{(r, p, q)}\right)$ and $\bar{D}_{x} L_{k+1}=W_{k}$.

Summarizing we have thus proved

Theorem 4.4 Let $W_{k} \in \mathcal{P}(m+1 ; k) \otimes \mathbb{R}_{\mathbb{R}} \mathbb{R}_{0, m+1}^{(r, p, q)}$. Then the following properties are equivalent:

(i) $W_{k} \in M T^{+}\left(m+1 ; k ; \mathbb{R}_{0, m+1}^{(r, p, q)}\right)$

(ii) there exists $L_{k+1} \in \mathcal{H}_{+}\left(m+1 ; k+1 ; \mathbb{R}_{0, m}^{(r, p, q)}\right)$ such that $W_{k}=\bar{D}_{x} L_{k+1}$ i.e. $\bar{D}_{x}: \mathcal{H}_{+}(m+$ $\left.1 ; k+1 ; \mathbb{R}_{0, m}^{(r, p, q)}\right) \rightarrow M T^{+}\left(m+1 ; k ; \mathbb{R}_{0, m+1}^{(r, p, q)}\right)$ is surjective.

By virtue of Theorem 4.4 and Proposition 4.2 we obtain

Proposition 4.5(i) Let $r$ be even. If $m+1$ is even and $r+2 q=m+1$ or $m+1$ is odd and $r+2 q=m$, then

$$
\overline{D_{x}}: \mathcal{H}\left(\mathbb{R}^{m+1} ; k+1 ; \mathbb{R}_{0, m}^{(r, p, q)}\right) \longrightarrow M T^{+}\left(\mathbb{R}^{m+1} ; k ; \mathbb{R}_{0, m+1}^{(r, p, q)}\right)
$$

is surjective.

$$
(i i) \overline{D_{x}}: \mathcal{H}\left(\mathbb{R}^{m+1} ; k+1 ; \mathbb{R}_{0, m}^{+}\right) \longrightarrow M T^{+}\left(\mathbb{R}^{m+1} ; k ; \mathbb{R}_{0, m+1}^{+}\right)
$$

is surjective.

\subsection{Primitives}

In this section we solve the following problem:

(P.3) Let $W_{k} \in M T^{+}\left(m+1 ; k ; \mathbb{R}_{0, m+1}^{(r, p, q)}\right)$ be given. Find $W_{k+1} \in$ $M T^{+}\left(m+1 ; k+1 ; \mathbb{R}_{0, m+1}^{(r, p, q)}\right)$ such that $W_{k}=\bar{D}_{x} W_{k+1}$.

Notice that in [6] and [13] it was proved that for each $P_{k} \in M^{+}(k), M^{+}(k)$ being the space of $\mathbb{R}_{0, m+1^{-}}$ valued left monogenic homogeneous polynomials of degree $k$ in $\mathbb{R}^{m+1}$, there exists $P_{k+1} \in M^{+}(k+1)$ such that $P_{k}=\bar{D}_{x} P_{k+1}$.

The specific feature in answering the problem (P.3) obviously lies in the fact that primitivation may be realized between spaces of homogeneous polynomial solutions to a given (GMT)-system of type $(r, p, q)$. 
To this end, suppose that $W_{k} \in M T^{+}\left(m+1 ; k ; \mathbb{R}_{0, m+1}^{(r, p, q)}\right)$.

¿From Theorem 4.2, it follows that there exists $L_{k+1} \in \mathcal{H}_{+}\left(m+1 ; k+1 ; \mathbb{R}_{0, m}^{(r, p, q)}\right)$ such that $W_{k}=$ $\bar{D}_{x} L_{k+1}$.

By means of the construction worked out in section 4.1, we may associate with $L_{k+1}$ an element $L_{k+2}^{*} \in$ $\mathcal{H}_{+}\left(m+1 ; k+2 ; \mathbb{R}_{0, m}^{(r, p, q)}\right)$ such that $W_{k+1}^{*}=\bar{D}_{x} L_{k+2}^{*}=U_{k+1}+\bar{e}_{0} V_{k+1} \in M T^{+}\left(m+1 ; k+1 ; \mathbb{R}_{0, m+1}^{(r, p, q)}\right)$ with $U_{k+1}=\partial_{x_{0}} L_{k+2}^{*}=L_{k+1}$ and $V_{k+1}=-\partial_{\underline{x}} L_{k+2}^{*}$.

It is then easily checked that $W_{k}=\bar{D}_{x}\left(\frac{1}{2} W_{k+1}^{*}\right)$.

We thus have proved

Theorem 4.6 Let $W_{k} \in M T^{+}\left(m+1 ; k ; \mathbb{R}_{0, m+1}^{(r, p, q)}\right)$ be given. Then there exists $W_{k+1} \in M T^{+}(m+$ $\left.1 ; k+1 ; \mathbb{R}_{0, m+1}^{(r, p, q)}\right)$ such that $W_{k}=\bar{D}_{x} W_{k+1}$.

\section{The lower dimensional cases $\mathbb{R}^{3}$ and $\mathbb{R}^{4}$}

In this section we investigate the possibilities for (GMT)-systems of type $(r, p, q), r$ being even, in $\mathbb{R}^{3}$ and $\mathbb{R}^{4}$. In particular a method is described for constructing bases in the spaces $M T^{+}\left(4 ; k ; \mathbb{R}_{0,4}^{(r, p, q)}\right)$. As in each of the cases which occur, basic knowledge is used from the case $M T^{+}\left(3 ; k ; \mathbb{R}_{0,3}^{ \pm}\right)$, for convenience of the reader we recall in section 5.1 some properties of the latter space. For a full description we refer to [7].

5.1. The case $M T^{+}\left(3 ; k ; \mathbb{R}_{0,3}^{+}\right)$

Following (2.2), $\mathbb{R}_{0,3}$ may be decomposed as

$$
\mathbb{R}_{0,3}=\mathbb{R}_{0,3}^{+} \oplus \mathbb{R}_{0,3}^{-}
$$

where

$$
\mathbb{R}_{0,3}^{+}=\operatorname{span}_{\mathbb{R}}\left(1, e_{1} e_{2}, e_{2} e_{0}, e_{0} e_{1}\right)
$$

and

$$
\mathbb{R}_{0,3}^{-}=\operatorname{span}_{\mathbb{R}}\left(e_{0}, e_{1}, e_{2}, e_{0} e_{1} e_{2}\right)
$$

Notice hereby that $\mathbb{R}_{0,3}^{+} \cong \mathbb{H}$, the algebra of real quaternions.

As pointed out in section 2, right multiplication with the pseudo-sealar $e_{0} e_{1} e_{2}$ establishes an isomorphism between $\mathbb{R}_{0,3}^{-}$and $\mathbb{R}_{0,3}^{+}$, i.e.

$$
\mathbb{R}_{0,3}^{-}=\mathbb{R}_{0,3}^{+} e_{0} e_{1} e_{2}
$$


Consequently

$$
M T^{+}\left(3 ; k ; \mathbb{R}_{0,3}^{-}\right)=M T^{+}\left(3 ; k ; \mathbb{R}_{0,3}^{+}\right) e_{0} e_{1} e_{2} .
$$

In [7], it was proved that $\operatorname{dim}_{\mathbb{R}} M T^{+}\left(3 ; k ; \mathbb{R}_{0,3}^{+}\right)=4(k+1)$ and a method was elaborated for constructing a basis for $M T^{+}\left(3 ; k ; \mathbb{R}_{0,3}^{+}\right)$. As was also mentioned in [7], the space $M T^{+}\left(3 ; k ; \mathbb{R}_{0,3}^{+}\right)$is isomorphic to the space $M_{k}(\mathbb{H} ; \mathbb{R})$, the space of $\mathbb{H}$-valued homogeneous polynomial solutions of degree $k$ of the Fueter operator $D$ in $\mathbb{R}^{3}$. A method for constructing an orthormal basis for $M_{k}(\mathbb{H} ; \mathbb{R})$ was worked out in [4] and [11] (see also [12]).

Finally, let us recall that in $\mathbb{R}^{3}$, the only $(r, p, q)$-subspace occuring for $r$ even is the space $\mathbb{R}_{0,3}^{+}=$ $\mathbb{R}_{0,3}^{(0,0,1)}$.

\subsection{The spaces $M T^{+}\left(4 ; k ; \mathbb{R}_{0,4}^{(r, p, q)}\right), r$ even}

The possible types of (GMT)-systems of type $\mathbb{R}_{0,4}^{(r, p, q)}$ have been described in section 2 .

In the following subsections we shall work out methods for constructing bases for the spaces $M T^{+}\left(4 ; k ; \mathbb{R}_{0,4}^{(r, p, q)}\right)$.

\subsubsection{The case $\mathbb{R}_{0,4}^{+}$}

Let us first of all recall that if $e=\left(e_{0}, e_{1}, e_{2}, e_{3}\right)$ is an orthogonal basis of $\mathbb{R}^{0,4}$ generating the Clifford algebra $\mathbb{R}_{0,4}$, then $\underline{e}=\left(e_{1}, e_{2}, e_{3}\right)$ is an orthogonal basis of $\mathbb{R}^{0,3}$ which generates the Clifford algebra $\mathbb{R}_{0,3}$ inside $\mathbb{R}_{0,4}$. It thus follows that

$$
\mathbb{R}_{0,4}^{+}=\mathbb{R}_{0,3}^{+} \oplus \bar{e}_{0} \mathbb{R}_{0,3}^{-}
$$

where

$$
\mathbb{R}_{0,3}^{+}=\operatorname{span}_{\mathbb{R}}\left(1, e_{2} e_{3}, e_{3} e_{1}, e_{1} e_{2}\right)=\mathbb{R}_{0,3}^{(0,0,1)}
$$

and

$$
\mathbb{R}_{0,3}^{-}=\operatorname{span}_{\mathbb{R}}\left(e_{1}, e_{2}, e_{3}, e_{1} e_{2} e_{3}\right)=\mathbb{R}_{0,3}^{(1,0,1)}
$$

Notice hereby that $\mathbb{R}_{0,3}^{+} \cong \mathbb{H}=\mathbb{R} \oplus V e c t \mathbb{H}$ where $V e c t \mathbb{H}=\operatorname{span}_{\mathbb{R}}\left(e_{2} e_{3}, e_{3} e_{1}, e_{1} e_{2}\right)$ is ismorphic to the space of pure quaternions.

Hence, an element $W_{k} \in M T^{+}\left(4 ; k ; \mathbb{R}_{0,4}^{+}\right)$splits into

$$
W_{k}=U_{k}+\bar{e}_{0} V_{k}
$$

where $U_{k}$ and $V_{k}$ are harmonic polynomials of degree $k$ which are, respectively, $\mathbb{R}_{0,3}^{+}$and $\mathbb{R}_{0,3^{-}}^{-}$ valued. 


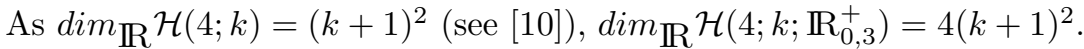

Now take a basis $\mathcal{S}_{k}=\left\{S_{k}^{(j)}: j=1, \ldots,(k+1)^{2}\right\}$ of $\mathcal{H}(4 ; k)$ and consider in

$\mathcal{H}(4 ; k+1)$ the linearly independent subset $\widetilde{\mathcal{S}}_{k+1}=\left\{\widetilde{S}_{k+1}^{(j)}: j=1, \ldots,(k+1)^{2}\right\}$, where for each $j=1, \ldots,(k+1)^{2}$ (see also $(4.6)$,

$$
\widetilde{S}_{k+1}^{(j)}(x)=\int_{0}^{x_{0}} S_{k}^{(j)}(t, \underline{x}) d t-\widetilde{h}_{k+1}^{(j)}(\underline{x})
$$

whith $\Delta_{\underline{x}} \widetilde{h}_{k+1}^{(j)}(x)=\partial_{x_{0}} S_{k}^{(j)}(0, \underline{x})$.

Furthermore, put $\widetilde{\mathcal{H}}_{k+1}=\widetilde{\mathcal{S}}_{k+1} \otimes\left\{1, e_{2} e_{3}, e_{3} e_{1}, e_{1} e_{2}\right\}$, where as usual for $S \in \widetilde{\mathcal{S}}_{k+1}$ and $\lambda \in\left\{1, e_{2} e_{3}, e_{3} e_{1}, e_{1} e_{2}\right\}, S \otimes \lambda$ is denoted by $S \lambda$. Then one may easily check that $\bar{D}_{x} \widetilde{\mathcal{H}}_{k+1}$ is a linearly independent subset of $M T^{+}\left(4 ; k ; \mathbb{R}_{0,4}^{+}\right)$.

Now consider again the element $W_{k} \in M T^{+}\left(4 ; k ; \mathbb{R}_{0,4}^{+}\right)$with $W_{k}=U_{k}+\bar{e}_{0} V_{k}$ (see also (5.2)) and lift up $U_{k} \in \mathcal{H}(4 ; k) \otimes \mathbb{R}_{\mathbb{R}} \mathbb{R}_{0,3}^{+}$to $\widetilde{U}_{k+1} \in \mathcal{H}(4 ; k+1) \otimes \mathbb{R} \mathbb{R}_{0,3}^{+}$, and this following the construction indicated above (see (5.3)).

Putting $\widetilde{W}_{k}=\bar{D}_{x} \widetilde{U}_{k+1}$, we have that $\widetilde{W}_{k} \in M T^{+}\left(4 ; k ; \mathbb{R}_{0,4}^{+}\right)$decomposes as

$$
\widetilde{W}_{k}=\widetilde{U}_{k}+\bar{e}_{0} \widetilde{V}_{k}
$$

where $\widetilde{U}_{k}=\partial_{x_{0}} \widetilde{U}_{k+1}=U_{k}$.

Consequently $\widetilde{W}_{k}-W_{k}=\bar{e}_{0}\left(\widetilde{V}_{k}-V_{k}\right)$ is $\bar{e}_{0} \mathbb{R}_{0,3}^{-}$-valued and left monogenic. This implies that $\widetilde{V}_{k}-V_{k}$ is independent of $x_{0}$ and that $\partial_{\underline{x}}\left(\widetilde{V}_{k}-V_{k}\right)=0$ or $\widetilde{V}_{k}-V_{k} \in M T^{+}\left(3 ; k ; \mathbb{R}_{0,3}^{-}\right)$.

But $M T^{+}\left(3 ; k ; \mathbb{R}_{0,3}^{-}\right)=M T^{+}\left(3 ; k ; \mathbb{R}_{0,3}^{+}\right) e_{1} e_{2} e_{3}, \quad e_{1} e_{2} e_{3}=e_{\stackrel{\circ}{\circ}}$ being the pseudo-scalar of $\mathbb{R}_{0,3}$. Choose a basis $\mathcal{B}_{k}$ for $M T^{+}\left(3 ; k ; \mathbb{R}_{0,3}^{+}\right)$(see secton 5.1 ).

Coming back to

$$
W_{k}=U_{k}+\bar{e}_{0} V_{k}
$$

we have that

$$
W_{k}=\tilde{W}_{k}+\bar{e}_{0}\left(V_{k}-\tilde{V}_{k}\right)
$$

where $\tilde{W}_{k} \in \operatorname{span}_{\mathbb{R}}\left(\bar{D}_{x} \widetilde{\mathcal{H}}_{k+1}\right)$ and $\bar{e}_{0}\left(V_{k}-\widetilde{V}_{k}\right) \in \operatorname{span}_{\mathbb{R}}\left(e_{0} \mathcal{B}_{k} e_{\stackrel{\circ}{ }}\right)$.

As for any $a \in \mathbb{R}_{0,3}^{+}, e_{0} a e_{\stackrel{\circ}{\circ}}=a e_{0} e_{{ }_{M}}=a e_{M}$, where $e_{M}=e_{0} e_{1} e_{2} e_{3}$ is the pseudo-scalar of $\mathbb{R}_{0,4}$, we obtain that $W_{k} \in \operatorname{span}_{\mathbb{R}}\left(\left(\bar{D}_{x} \widetilde{\mathcal{H}}_{k+1}\right) \cup\left(\mathcal{B}_{k} e_{M}\right)\right)$.

It may be easily verified that $\left(\bar{D}_{x} \widetilde{\mathcal{H}}_{k+1}\right) \cup\left(\mathcal{B}_{k} e_{M}\right)$ is linearly independent in $M T^{+}\left(4 ; k ; \mathbb{R}_{0,4}^{+}\right)$. As follows from the construction made above, it is also a generating set for that space, whence it is a basis for it. 
As $\widetilde{\mathcal{H}}_{k+1}$ contains $4(k+1)^{2}$ elements and $\mathcal{B}_{k}$ contains $4(k+1)$ elements, we have proved

\section{Theorem 5.1}

(i) $\operatorname{dim}_{\mathbb{R}^{M}} M T^{+}\left(4 ; k ; \mathbb{R}_{0,4}^{+}\right)=4(k+1)(k+2)$.

(ii) $\left(\left(\bar{D}_{x} \widetilde{\mathcal{H}}_{k+1}\right) \cup\left(\mathcal{B}_{k} e_{M}\right)\right)$ is a basis for $M T^{+}\left(4 ; k ; \mathbb{R}_{0,4}^{+}\right)$.

Remarks(1) The space $M T^{+}\left(4 ; k ; \mathbb{R}_{0,4}^{+}\right)$coincides with the so-called space of $\mathbb{R}_{0,4}^{+}$-valued inner spherical monogenics of degree $k$ in $\mathbb{R}^{4}$ (see [8]). It is well known that as a right module over the algebra $\mathbb{R}_{0,4}^{+}, \operatorname{dim}_{\mathbb{R}_{0,4}^{+}} M T^{+}\left(4 ; k ; \mathbb{R}_{0,4}^{+}\right)=\frac{(k+2)(k+1)}{2}$.

It follows that, as $\operatorname{dim}_{\mathbb{R}} \mathbb{R}_{0,4}^{+}=8, \operatorname{dim}_{\mathbb{R}} M T^{+}\left(4 ; k ; \mathbb{R}_{0,4}^{+}\right)=4(k+2)(k+1)$, thus confirming the result obtained in Theorem 5.1 (i).

A classical basis for the right $\mathbb{R}_{0,4}^{+}$-module $M^{+}\left(4 ; k ; \mathbb{R}_{0,4}^{+}\right)$is given by the set $B_{k}$ of so-called Fueter polynomials:

$$
B_{k}=\left\{V_{\underline{\alpha}}: \underline{\alpha} \in \mathbb{N}^{3},|\underline{\alpha}|=k\right\}
$$

where for $\underline{\alpha}=\left(\alpha_{1}, \alpha_{2}, \alpha_{3}\right)$,

$$
V_{\underline{\alpha}}(x)=\sum_{j=0}^{|\underline{\alpha}|}(-1)^{j} \frac{x_{0}^{j}}{j !}\left(\bar{e}_{0} \partial_{\underline{x}}\right)^{j}(\underline{x} \underline{\underline{\alpha}})
$$

with $\underline{x} \underline{\underline{\alpha}}=x_{1}^{\alpha_{1}} x_{2}^{\alpha_{2}} x_{3}^{\alpha_{3}}$.

It thus follows that

$$
B_{k}^{*}=\left\{V_{\underline{\alpha}}, V_{\underline{\alpha}} \bar{e}_{0} e_{1}, V_{\underline{\alpha}} \bar{e}_{0} e_{2}, V_{\underline{\alpha}} \bar{e}_{0} e_{3}, V_{\underline{\alpha}} e_{1} e_{2}, V_{\underline{\alpha}} e_{1} e_{3}, V_{\underline{\alpha}} e_{2} e_{3}, V_{\underline{\alpha}} e_{0} e_{1} e_{2} e_{3}: \underline{\alpha} \in \mathbb{N}^{3},|\underline{\alpha}|=k\right\}
$$

is a basis for the real vector space $M T^{+}\left(4 ; k ; \mathbb{R}_{0,4}^{+}\right)$.

(2) By means of Theorem 4.2 and the Remark following it, we have that $\bar{D}_{x}: \mathcal{H}\left(4 ; k+1 ; \mathbb{R}_{0,3}^{+}\right) \rightarrow$

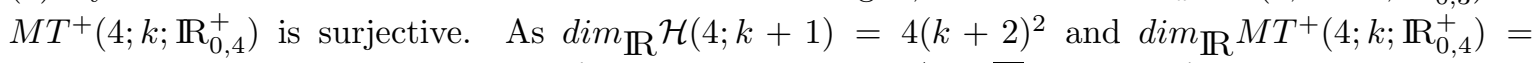
$4(k+2)(k+1)$, we have that $\operatorname{dim}_{\mathbb{R}}\left\{H_{k+1} \in \mathcal{H}\left(4 ; k+1 ; \mathbb{R}_{0,3}^{+}\right): \bar{D}_{x} H_{k+1}=0\right\}$

$=4(k+2)$.

\subsubsection{The case $\mathbb{R}_{0,4}^{(2)} \oplus \mathbb{R}_{0,4}^{(4)}$}

We apply the same procedure as worked out in the foregoing subsection.

Let $W_{k} \in M T^{+}\left(4 ; k ; \mathbb{R}_{0,4}^{(2)} \oplus \mathbb{R}_{0,4}^{(4)}\right)$ and write it out as

$$
W_{k}=U_{k}+\bar{e}_{0} V_{k}
$$


where $U_{k}$ and $V_{k}$ are harmonic homogeneous polynomials of degree $k$ in $\mathbb{R}^{4}$ which are respectively, $\mathbb{R}_{0,3}^{(2)} \oplus \mathbb{R}_{0,3}^{(4)}$ and $\mathbb{R}_{0,3}^{(1)} \oplus \mathbb{R}_{0,3}^{(3)}=\mathbb{R}_{0,3}^{-}$-valued. As $\mathbb{R}_{0,3}^{(4)}=\{0\}$, we have that $U_{k}$ is $\mathbb{R}_{0,3}^{(2)}$-valued with $\mathbb{R}_{0,3}^{(2)}=\operatorname{span}_{\mathbb{R}}\left(e_{2} e_{3}, e_{3} e_{1}, e_{1} e_{2}\right) \cong V e c t \mathbb{H}$.

Starting again from a basis $\mathcal{S}_{k}$ of $\mathcal{H}(4 ; k)$, lifting it up by means of $(5.3)$ to $\widetilde{S}_{k+1}$ and putting $\widetilde{\mathcal{H}}_{0, k+1}=\widetilde{\mathcal{S}}_{k+1} \otimes\left\{e_{2} e_{3}, e_{3} e_{1}, e_{1} e_{2}\right\}$ we obviously have that $\widetilde{\mathcal{H}}_{k+1}=\widetilde{\mathcal{S}}_{k+1} \cup \widetilde{\mathcal{H}}_{0, k+1}$.

The reasoning made in the foregoing subsection then leads to

\section{Theorem 5.2 .}

(i) $\left(\bar{D}_{x} \widetilde{\mathcal{H}}_{0, k+1}\right) \cup\left(\mathcal{B}_{k} e_{M}\right)$ is a basis for $M T^{+}\left(4 ; k ; \mathbb{R}_{0,4}^{(2)} \oplus \mathbb{R}_{0,4}^{(4)}\right)$

(ii) $\operatorname{dim}_{\mathbb{R}} M T^{+}\left(4 ; k ; \mathbb{R}_{0,4}^{(2)} \oplus \mathbb{R}_{0,4}^{(4)}\right)=(k+1)(3 k+7)$.

Remark. As already mentioned, $\mathbb{R}_{0,3}^{(4)}=\{0\}$. From Theorem 4.2 it then follows that $\bar{D}_{x}: \mathcal{H}(4 ; k+$ $\left.1 ; \mathbb{R}_{0,3}^{(2)}\right) \rightarrow M T^{+}\left(4 ; k ; \mathbb{R}_{0,4}^{(2)} \oplus \mathbb{R}_{0,4}^{(4)}\right)$ is surjective.

5.2.3 The case $\mathbb{R}_{0,4}^{(0)} \oplus \mathbb{R}_{0,4}^{(2)}$

Notice that $\mathbb{R}_{0,4}^{(0)} \oplus \mathbb{R}_{0,4}^{(2)}=\left(\mathbb{R}_{0,4}^{(2)} \oplus \mathbb{R}_{0,4}^{(4)}\right) e_{M}$.

We so have

Theorem 5.3

(i) $\left(\bar{D}_{x}\left(\widetilde{\mathcal{H}}_{0, k+1} e_{M}\right)\right) \cup \mathcal{B}_{k}$ is a basis for $M T^{+}\left(4 ; k ; \mathbb{R}_{0,4}^{(0)} \oplus \mathbb{R}_{0,4}^{(2)}\right)$

(ii) $\operatorname{dim}_{\mathbb{R}^{M T}} M T^{+}\left(4 ; k ; \mathbb{R}_{0,4}^{(0)} \oplus \mathbb{R}_{0,4}^{(2)}\right)=(k+1)(3 k+7)$.

\section{Received: March 2009. Revised: April 2009.}

\section{References}

[1] R. Abreu Blaya, J. Bory Reyes, R. Delanghe and F. Sommen, Generalized MoisilThéodoresco systems and Cauchy integral decompositions, Int. J. Math. Math. Sci., Vol. 2008, Article ID746946, 19 pages.

[2] F. Brackx and R. Delanghe, On harmonic potential fields and the structure of monogenic funtions, Z. Anal. Anwendungen 22 (2003) 261-273. Corrigendum to: Z. Anal. Anwendungen 25 (2006) 407-410.

[3] F. Brackx, R. Delanghe and F. Sommen, Differential forms and / or multi-vector functions, CUBO 7 (2005) 139-170.

[4] I. CAÇÃo, Constructive approximation by monogenic polynomials, (Ph. D-thesis, Universidade de Aveiro, 2004). 
[5] A. Cialdea, On the theory of self-conjugate differential forms, Atti del Seminario Matematico e Fisico dell' Universit di Modena 46 (1998) 595-620.

[6] R. Delanghe, On primitives of monogenic funtions, Complex Variables and Elliptic Equations 51 (2006) 959-970.

[7] R. Delanghe, On homogeneous polynomial solutions of the Moisil-Théodoresco system in $\mathbb{R}^{3}$, Computational Methods and Function Theory 9 (2009) 199-212.

[8] R. Delanghe, F. Sommen and V. Souček, Clifford algebra and Spinor-Valued Functions (Kluwer, Dordrecht, 1992).

[9] A. Dzhuraev, Methods of singular equations (Longman, 1992, Harlow, 1992).

[10] G. Folland, Introduction to Partial Differential Equations (Princeton Univ. Press, Princeton, 1976).

[11] K. Gürlebeck and I. CaÇÃO, Monogenic primitives of monogenic functions, in : Simos, T.E. (ed.); Tsitouras, Ch. (ed.): ICNAAM 2004, International conference on numerical analysis and applied mathematics 2004, Chalkis, Greece, September 10-14, 2004.

[12] K. Gürlebeck, K. Habetha and W. Spressig, Holomorphic functions in the plane and n-dimensional space (Birkhäuser Verlag, Basel, 2007)

[13] K. GüRlebeck And H. MaloneK, A hypercomplex derivative of monogenic functions in $\mathbb{R}^{n+1}$ and its applications, Complex Variables 39 (1999) 199-228.

[14] K. Gürlebeck and W. Spressig, Quatermonic Analysis and Elliptic Boundary Value Problems (Akademie-Verlag, Berlin, 1989).

[15] V. Kravchenko and M. Shapiro, Integral Respresentations for Spatial Models of Mathematical Physics, Pitman Research Notes in Mathematics Series 351 (Longman, Harlow, 1996).

[16] K. Maurin, Analysis, part II (Reidel Publishing Company, Dordrecht-Boston-London, PWNPolish Scientific Publishers, Warszawa, 1980).

[17] Gr. Moisil and N. ThÉodoresco, Fonctions holomorphes dans l'espace, Mathematica Cluj 5 (1931) 142-159.

[18] C. Nolder, Conjugate harmonic functions and Clifford algebras, J. Math. Anal. Appl. 302 (2005) 137-142.

[19] E. Stein And G. Weiss, On the theory of harmonic functions of several variables, I. The Theory of $H^{p}$-spaces, Acta Mathematica 103 (1960) 25-62.

[20] E. Stein And G. Weiss, Generalization of the Cauchy-Riemann equations and representations of the rotation group, Amer. J. Math. 90 (1968) 163-196. 